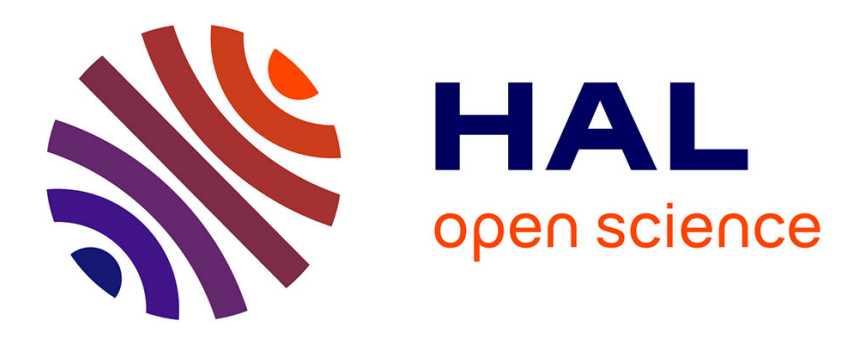

\title{
Système électoral et révolution : la voie tunisienne Eric Gobe
}

\section{To cite this version:}

Eric Gobe. Système électoral et révolution: la voie tunisienne. Pouvoirs - Revue française d'études constitutionnelles et politiques, 2016, La Tunisie, 156, 10.3917/pouv.156.0071 . halshs-01500082

\section{HAL Id: halshs-01500082 \\ https://shs.hal.science/halshs-01500082}

Submitted on 1 Apr 2017

HAL is a multi-disciplinary open access archive for the deposit and dissemination of scientific research documents, whether they are published or not. The documents may come from teaching and research institutions in France or abroad, or from public or private research centers.
L'archive ouverte pluridisciplinaire HAL, est destinée au dépôt et à la diffusion de documents scientifiques de niveau recherche, publiés ou non, émanant des établissements d'enseignement et de recherche français ou étrangers, des laboratoires publics ou privés. 


\section{Système électoral et révolution : la voie tunisienne}

Eric Gobe, directeur de recherche au CNRS, Institut de recherche sur le Maghreb contemporain de Tunis (USR 3077)

Après le départ du président Ben Ali du pouvoir le 14 janvier 2011, sous la pression des mouvements populaires de protestation, les différents acteurs impliqués dans le processus « révolutionnaire » 1 ont opté pour l'écriture d'une nouvelle Constitution etl'élection d'une Assemblée nationale constituante (ANC). Ce scénario, qui implique la rédaction d'une loi électorale spécifique, est principalement écrit par trois institutions de transition : le président de la République par intérim,le gouvernement ${ }^{2}$ etl'Instance supérieure pour la réalisation des objectifs de la révolution, de la réforme politique et de la transition démocratique (ISROR) ${ }^{3}$. C'est cette dernière institutioncomposée de membres nommés,censés représenter les principaux courants politiques et forces vives de la société tunisienne ${ }^{4}$,qui doit en priorité concrétiser l'un des principaux «objectifs de la révolution»: élaborer une législation électorale permettantau « peuple révolutionnaire »d'exprimer sa volonté souveraine à travers l'élection de l'ANC ${ }^{5}$.

Aussi les membres de l'ISRORs'interrogent-ils sur la loi électorale qui permettrait la meilleure adéquation possible entre «peuple révolutionnaire » et peuple électeur.Cequestionnementest repris, pour partie, par l'ANC qui, suite à l'adoption le 26 janvier 2014 d'une nouvelle Constitution prévoyant l'élection d'une Assemblée des représentants du peuple et d'un président de la République au suffrage universel direct à la majorité absolue des suffrages exprimés, doit élaborer une nouvelle loi électorale. Après plus de deux ans de crises, la dynamique politique et la logique de compromis privilégiée par les partis incitent l'ANCà reconduire largement, pour le scrutin législatif, le système électoralqui avait prévalu en 2011 pour son élection.

In fine, confrontés à la question du caractère soluble du «peuple révolutionnaire » dans le «peuple électeur », les représentants nommés (ISROR) et les constituants élus (ANC) ont tenté d'y répondre en adoptant un mode de scrutin proportionnel (I), en affirmant la nécessaire surreprésentation de certaines catégories de la population (II) et en proposant l'exclusion du corps des représentants du peuple, des citoyens considérésindignes d'y participer, car soutiens du président déchu(III).

\section{Le peuple révolutionnaire est-il soluble dans le peuple électeur?}

\footnotetext{
${ }^{1}$ La majeure partie des acteurs politiques et sociaux tunisiens ont désigné, au lendemain du 14 janvier 2011, sous le vocable de révolution,la période couverte par les principaux mouvements protestataires entre décembre 2010 et janvier, voire février 2011. Voir K. Bendana «Entrer dans l'histoire deRévolution tunisienne ? », L'Année du Maghreb 2014, Paris, CNRS Editions, 2014, p. 49-58.

${ }^{2}$ Les deux sont dotés de larges attributions législatives exercées par voie de décrets-lois.

${ }^{3}$ Selon le décret-loi la régissant, l'ISROR est « chargée d'étudier les textes législatifs ayant trait à l'organisation politique et de proposer les réformes à même de concrétiser les objectifs de la révolution, relatifs au processus démocratique », Journal officiel de la République tunisienne (JORT), « Décret-loi n 6 du 18 février 2011 relatif à la création de l'ISROR (en arabe) », $\mathrm{n}^{\circ} 13,1^{\mathrm{er}}$ mars 2011, p. 200.

${ }^{4}$ Son assemblée est formée « de personnalités politiques nationales, de représentants des partis politiques, des instances, des organisations, des associations et des composantes de la société civile concernées par les affaires nationales dans la capitale et les régions, parmi ceux qui ont participé à la révolution ou l'ont soutenue, qui seront nommés par arrêté du Premier ministre sur proposition des organismes concernés » (art. 3 du décret-loi). Par ailleurs, un comité d'expertise composé d'universitaires tunisiens spécialistes de droit public, bilingues français arabe, imprégnés de culture juridique des droits de l'Homme, est chargé de rédiger les textes soumis à l'assemblée pour approbation. VoirJORT, op. cit.

${ }^{5}$ J.-P. Bras, «Le peuple est-il soluble dans la constitution? Leçons tunisiennes », L'Année du Maghreb 2012, Paris, CNRS Editions, 2012, p. 103-119.
} 
Deux problématiques ont structuré les débats sur le choix du mode de scrutin au sein de l'ISROR : la première, posée de manière explicite, a concerné, comme sous d'autres cieux, la question de la meilleure manière de représenter le «peuple», alors que la seconde, formuléeimplicitement, se rapportait plus spécifiquement àl'ampleur de la représentativité du parti incarnant l'islam politique dans la société tunisienne, le Mouvement Ennahdha.

Tout au long des débats, l'ISROR s'est interrogée sur les conditions d'une bonne représentation du peuple révolutionnaire. Son approche s'est largement inscrite dans la perspective sociologique de la «représentation-figuration»: il s'est agi de définir les particularités sociales les plus pertinentes au regard du soulèvement populaire afin que l'ANC « les reproduise à une échelle réduite ${ }^{6}$. À cet égard, le mode de scrutin proportionnel de liste est apparu comme le mieux à même de réaliser l'idéal d'un système représentatif justesupposé refléter «les caractéristiques de la structure sociale ${ }^{7}$.

Si le scrutin majoritaire a trouvé quelques adeptesau sein de l'ISROR $^{8}$, la majorité de sesmembres lui reproche d'avoir le principal inconvénient d'activer les «travers » de la société tunisienne. Porte ouverte à l'expression des clivages primordiaux, il favoriserait les notables, les relations clientélistes, la corruption et son corollaire l'achat de voix. Lescitoyens tunisiens, imprégnés de «clanisme et de régionalisme », enverraient à l'ANC desdéputés incapables de porter des programmes politiques, car fortement encastrés dans des logiques sociales locales 9 .

Toutefois, le consensus affiché sur le mode de scrutin de proportionnel de liste par les membres de l'ISROR est lourd de sous-entendus. En effet, il reste à choisir la modalité d'attribution des sièges «en l'air " ${ }^{10}$ selon le plus fort reste ou la plus forte moyenne.Les partisans de la première option sont conscients qu'elle avantage les petites listes. Elle apparait,pour les membres de la gauche radicale et les représentants des courants sécularistes tunisiens,comme un antidote à la vocation hégémonique d'Ennahdha. Anouar Kousri, représentant de la Ligue tunisienne de défense des droits de l'Homme et membre du Parti communiste des ouvriers de Tunisie, mouvementd'extrême gauche, composée d'une poignée de militants, dénonce le mode de scrutin proportionnel à la plus forte moyenne comme « injuste» «avantageant les deux listes arrivées en tête » qui «pourraient prendre $80 \%$ des sièges $»^{11}$.

En revanche, le Mouvement Ennahdha, parti de masse, certain de sa réussite aux élections de la future ANC, opte non seulementpour un mode de scrutin proportionnel à la plus forte moyenne, mais aussi pour l'introduction d'un seuil de $5 \%$ des suffrages exprimés en dessous duquel une liste n'est pas prise en compte dans le calcul de la répartition des sièges ${ }^{12}$.

\footnotetext{
${ }^{6}$ À la «représentation-figuration» s'oppose «la représentation-mandat» qui «ne conçoit qu'une égalité purement quantitative, visant à ce que la souveraineté soit mécaniquement partagée entre tous les citoyens ». VoirP.Rosanvallon, Le peuple introuvable, Paris, Gallimard, 1998, p. 179-180.

${ }^{7}$ Y.Meny et M.Sadoun, «Conception de la représentation et représentation proportionnelle », Pouvoirs, $\mathrm{n}^{\circ} 32$, 1985, p. 7 ; voir également B. Manin, Principes du gouvernement représentatif, Paris, Flammarion, 2012, p. 143148.

${ }^{8}$ Ils y ont vu un instrument susceptible de créer un lien personnel entre électeurs et élus et, par conséquent, de favoriser les candidats ancrés dans le terroir local.

${ }^{9}$ République tunisienne, Recueil des débats de l'ISROR (en arabe), (ci-après ISROR), Séance du 7 avril 2011, 2012, p. 148 et 154.

${ }^{10}$ J.-M.-A. Paroutaud, «Quelques réflexions théoriques sur la représentation proportionnelle », Revue française de science politique, vol. 13, $\mathrm{n}^{\circ}$ 3, 1963.

${ }^{11}$ ISROR, Séance du 7 avril,p. 189-190.

${ }^{12}$ ISROR, Séance du 4 avril, p. 134.
} 
Incapables de trouver un consensus sur le mode de scrutin, les membres de l'Instance votent pour adopter au final une attribution des sièges en l'air au plus fort reste (76 voix pour contre 51en faveur de la plus forte moyenne) $)^{13}$.

La question de l'établissement d'un seuil électoral reviendra avec force en 2014 lors des débats à 1'ANC sur la nouvelle loi relative aux élections et référendums. La commission de législation générale chargée de rédiger une première mouture du texte reprend à son compte la proposition formulée par plusieurs associations de promotion de la citoyenneté ${ }^{14}$ d'introduire un seuil de $3 \%$ des suffrages exprimés par circonscription,en dessous duquel une liste n'est pas prise en compte dans le calcul de la répartition des sièges ${ }^{15}$. L'objectif affiché est de réduire certains inconvénients de la représentation proportionnelle, notamment la fragmentation de l'offre politique et la déperdition des voix, qui risquent de déboucher sur la formation de gouvernements de coalition instables, sans base parlementaire suffisante ${ }^{16}$.

Mais le seuil de $3 \%$ est loin de faire l'unanimité.Les petits partis et élus « indépendants »,conscients de leur faible audience populaire et, par conséquent, de leurs difficultés à faire élire leurs représentants ont exprimé leur rejet de l'introduction de cette disposition dans le texte à soumettre au vote de l'ANC $^{17}$. Quant aux deux grands partis (Ennahdha et NidaaTounes ${ }^{18}$ ), ils voient dans le statu quole meilleur moyenqu'aucun des deux ne gouverne seul après les élections législatives ${ }^{19}$.

Toutefois, les effets de l'absence de seuil sont de moindre ampleur pour le scrutin du 26 octobre 2014 que pour celui du 23 octobre 2011.En raison de l'évolution desrapports de force politique depuis 2012, le nombre de suffrages perdus est en nette baisse (418 000 voix)et lesystème partisan tunisiena tendance à se concentrer : en 2011, 19 partis et 8 listes indépendantes étaient représentés à l'ANC, alors qu'en 2014, 14 partis, un front, et 3 listes indépendantes ont obtenu des sièges ${ }^{20}$.

La recomposition de la scène politique qui,depuis 2012,s'est structurée autour de deux principaux partis,l'un plutôt islamo-conservateur ou islamiste, incarné par Ennahdha, et l'autre,plutôt séculariste,représenté parNidaaTounes a contribué à créer un phénomène de vote utile en faveur de ses deux formations. Toutefois, cette bipolarisation politique relative est entravée parle mode de scrutin qui rend impossible l'émergence d'une majorité absolue au profit d'un parti.

\footnotetext{
${ }^{13}$ ISROR, Séance du 11 avril, p. 235.

${ }^{14}$ Centre citoyenneté, Jeunes sans frontières, Fondation for the future, Avant-projet de loi électorale, téléchargeable sur le site de Marsad, <http://majles.marsad.tn/fr/docs/53149a1f12bdaa6c2fc00dd6>.

${ }^{15} \mathrm{ANC}$, Rapport de la commission de législation générale concernant la proposition de loi organique relative aux élections et référendums (en arabe), mars 2014.

${ }^{16} I d$. Les élections de l'ANC, le 23 octobre 2011, ont d'ailleurs été marquées par un fort éparpillement des suffrages, puisque la multiplication des candidatures indépendantes a abouti à l'évaporation de près de 1300000 voix (32\% des suffrages exprimés) qui se sont portées sur des listes n'ayant envoyé aucun représentant à l'ANC. ${ }^{17}$ Marsad, «La commission de législation générale : début des débats autour de la proposition de loi électorale (en arabe) », Chroniques, 13 février 2014, <http://majles.marsad.tn/chroniques/5302197212bdaa52450a7791>

${ }^{18}$ Fondé en juin 2012 par le très bourguibien Béji Caïd Essebsi ( 88 ans), NidaaTounes s'est posé comme un fédérateur de toutes les sensibilités politiques tunisiennes anti-Ennahdha. Le mouvement ratisse large rassemblant en son sein des figures politiques proches de feu Habib Bourguiba, des caciques du parti dissous du président déchu, le Rassemblement constitutionnel démocratique, des patrons inquiets pour la bonne marche de leurs affaires, mais aussi des anciens militants de gauche.

${ }^{19}$ H. Zbiss, « Mode de scrutin : la bataille pour la démocratie a commencé », Réalités on line, 24 février 2014, <http://www.realites.com.tn/2014/02/mode-de-scrutin-la-bataille-pour-la-democratie-a-commence/>

${ }^{20}$ Mission d'observation électorale de l'Union européenne, Tunisie. Rapport final sur les élections législatives et présidentielles 2014, <http://eeas.europa.eu/eueom/missions/2014/tunisia/pdf/150313-rapport-final-moeuetunisie-2014_fr.pdf $>$.D'autres indicateurs rendent compte du fort éparpillement des voix lors des élections de 2011. Seules 6 listes ont obtenu plus de $3 \%$ des suffrages exprimés. Huit listes indépendantes et 8 partis ont eu un seul siège.
} 
Ainsi, si les résultats électoraux du 26 octobre 2014 scellent la victoire de NidaaTounes avec 37,56 \% des voix et 86 des 217 sièges à l'Assemblée des représentants du peuple (ARP) et placent Ennahdha en deuxième position avec 27,79 \% des suffrages exprimés et 69 sièges (contre 89 en 2011), aucun de ces deux partis ne disposede la majorité absolue des 109 députés nécessaires pour obtenir le vote de confiance à l'Assemblée ${ }^{21}$.

Par-delà la question du mode de scrutin idéal pour la Tunisie, le désir d'homothétie entre le «peuple révolutionnaire» et la représentation s'est prolongé dans la volonté d'instaurer, tant au sein de l'ISROR que de l'ANC, une «proportionalisationaccrue ${ }^{22} \mathrm{de}$ certaines catégories de population.

\section{Instaurer une proportionnalisation accrue}

Cette volonté d'introduire des distorsions de représentativité s'est d'abord focalisée sur le découpage des circonscriptions électorales. Elle renvoie aux questions des inégalités régionales en matière de peuplement et de marginalisation de certaines régions par le pouvoir central autoritaire. Dans l'esprit des membres de l'ISROR, lesgouvernorats(l'équivalent de nos préfectures) victimes de politiques publiques qui ont contribué à les vider de leurs habitants, doivent bénéficier d'une représentativitéaccrue à l'ANC ${ }^{23}$.

Par ailleurs, le président de l'Instance suggère que les listes comprennent des candidats provenant de délégations différentes (l'équivalent d'une sous-préfecture). "La révolution tunisienne [étant] celle de la jeunesse ${ }^{24}$, au moins l'un des candidats devra être âgé de moins de30 ans. Mais, cette disposition ne suffit pas à garantir la présence des moins de 30 ans à l'ANC puisque le texte n'impose pas de placer un jeune en tête de liste.

Le jeunisme révolutionnaire s'exprime également dans la disposition,présente dans les textes de 2011 et 2014, prévoyant l'élection du candidat le plus jeune, et non le plus âgé, en cas d'égalité des restes entre deux ou plusieurs listes. Mais par-delà le discours sur la reconnaissance du rôle joué par la jeunesse pendant la révolution, les petits partis dont les cadres et les militants sont relativement âgés ne disposent pas d'un vivier suffisant de jeunes candidats potentiels qu'ils pourraient intégrer à leur liste. Alors que la Commission de législation générale proposait qu'un candidat de moins de 30 ans figure impérativement parmi les trois premiers candidats de la liste, la Commission des consensus de l'ANC a réduit la portée de la mesure en prévoyant que chaque liste comporte parmi les 4 premiers noms « un candidat jeune, ne dépassant pas les 35 ans $» 25$.

Quant à la représentation des femmesparmi les élus, elle apparaît bien plus connectée à la problématique de l'égalité des sexes et de la parité qu'à la « révolution ». À l'ISROR, ellea été portée par deux associations féministes sécularistesnées sous la présidence Ben Ali, l'Association tunisienne des femmes démocrates et l'Association des femmes tunisiennes pour la recherche et le développement dont l'objectif est de faire en sorte que la loi électorale

\footnotetext{
${ }^{21}$ Selon la projection effectuée par le constitutionnaliste Ch.Gaddès, il aurait fallu instaurer un seuil de $5 \%$ pour que NidaaTounès obtienne la majorité absolue des sièges à l'ARP.Voir «Élections législatives du 26 octobre 2014. Que veulent dire les résultats ? », Réalités, 6 novembre 2014.

${ }^{22}$ Y.Mény et Y.Surel, Politique comparée, Paris, Montchrestien, 2009 ( 8 édition), p. 185.

${ }^{23}$ En attribuant un minimum de quatre députés, aux gouvernorats les moins peuplés (Tozeur, Kebili, Tataouine, Zaghouan), l'ISROR leur permet d'être des régions surreprésentées à l'ANC.

${ }^{24}$ Intervention de FadhelBettaher, représentant des régions, ISROR, Séance du 31 mars, p. 109.

${ }^{25}$ Le texte prévoit que les listes ne respectant pas cette disposition seront privées « de la moitié de la valeur totale de l'indemnité de financement public ». Décision de la Commission des consensus se rapportant à la représentativité des jeunes sur les listes. <https://www.facebook.com/AlBawsala/photos/pcb.753824274650401/753824237983738/?type=1\&permPage= $1>$.
} 
permette de représenter de manière arithmétique l'égalité hommes/femmes ${ }^{26}$. L'ensemble des membres de l'Instance étant d'accord pour introduire des quotas plus ou moins élevés de femmes sur les listes électorales, cela conduit le comité des experts juridiques de l'ISROR à élaborer deux propositions : la première prévoit que «Les candidatures sont présentées selon le principe de la parité entre femmes et hommes. Les candidats sont classés entre femmes et hommes dans les listes de manière alternée », alors que la seconde dispose que « chaque liste doit présenter au minimum $25 \%$ de femmes présentée de manière alternée ${ }^{27}$.Cette proposition d'instituer un quota est rejetée, alors que la première remporte les deux tiers des suffrages. Cette forme de parité dite verticale ${ }^{28} \mathrm{n}$ 'a pas pour autant signifié une égale représentation des hommes et des femmes à l'ANC : $93 \%$ des listes présentes aux élections du 23 octobre 2011 avaient un homme pour tête de liste. Sur les 217 sièges de l'ANC, 49 sont revenus à des femmes dont 42dupartiEnnahdha ${ }^{29}$. La forte présence de femmes du mouvement islamiste à l'ANC s'explique par l'ampleur de sa victoire électorale : alors qu'il n'avait qu'une femme tête de liste, Ennahdha est le seul parti à avoir remporté au moins 2 sièges dans chaque circonscription.

La consécration par la nouvelle Constitution dans son article 46du principe de la parité au sein des conseils élus fait espérer à certaines associations féministes que la prochaine loi électorale comprendra le principe de la parité horizontale ${ }^{30}$.

Mais au sein de la Commission de législation générale, les constituants n'approuvent pas tous ledit principe. Ils proposent une parité verticale avec un quotad'un tiers des femmes en tête de liste ${ }^{31}$. La Commission des consensus mentionne, quant à elle, un quota minimum de $30 \%$ de femmes en tête de liste avec sanction de privation de la moitié du financement public en cas de non-respect de ce principe. L'article ainsi rédigé est rejeté par une majorité relative des constituants,alors que la parité horizontale n'atteint pas le quorum de la majorité absolue, bien qu'approuvée par une majorité relative. Le résultat des votes en plénière signifie que les constituantsn'ont pas voulu assumer un refus clairement affiché de la parité horizontale. Ils se sont contentés, à une large majorité ${ }^{32}$, de reconduire le dispositif de 2011 de parité verticale.

Le nombre de femmes élues a tout de même progressé par rapport aux élections de 2011passant de 49 à 68. La concentration du vote sur les deux principaux partis de la scène politique explique l'essentiel de cet accroissement. Outre les 12 femmes élues comme tête de liste, les 56 autres députées proviennent exclusivement de NidaaTounes (32) et d'Ennahdha (24) qui ont obtenu suffisamment de voix pour faire élire les deuxièmes, quatrièmes, voire cinquièmes de leurs listes en vertu du principe de parité verticale ${ }^{33}$.

$\mathrm{Si}$ au regard des principes d'une juste représentation, il s'est agi pour le législateur tunisien d'élaborer une loi électorale permettant aux représentantsde la population d'être l'expression fidèle du corps social tunisien, il a également souhaité interdire, tout au moins en

\footnotetext{
${ }^{26}$ ISROR, Séancedu 4 avril, p. 135.

${ }^{27}$ ISROR, Séance du 11 avril, p. 234.

${ }^{28}$ Dans le cadre d'une parité verticale avec alternance, les listes de candidats comportent autant de femmes que d'hommes de sorte que si la tête de liste est un homme, le suivant est une femme, et vice-versa. Quant à la parité horizontale, elle assure une présence féminine plus forte dans les assemblées élues que la parité verticale avec alternance, puisque les listes de candidats, présentés par les mêmes partis politiques doivent avoir le même nombre de têtes de liste au féminin qu'au masculin.

${ }^{29}$ Ch.Gaddès, op. cit.

${ }^{30}$ Al Hunfington Post, «Tunisie: Des associations réclament la parité verticale et horizontale dans la loi électorale », <http://www.huffpostmaghreb.com/2014/04/23/tunisie-parite-loi-electo_n_5199135.html>.

${ }^{31} \mathrm{ANC}$, op. cit.

${ }^{32}$ Voir le site de Marsad qui répertorie les votes des députés :160 voix pour, 11 contre et 10 abstentions. Consultable sur <http://majles.marsad.tn/fr/vote/53623a3812bdaa078ab8250f >.

${ }^{33}$ Ch.Gaddès, op. cit.
} 
2011, les cadres de l'ancien régime, vus comme un danger pour la révolution et la transition démocratique, de se présenter aux élections.

\section{Rendre inéligible les citoyens « indignes »}

Les débats relatifs l'étendue de l'exclusion du droit de candidature des responsables de l'ancien régime ont été forts longs et lourds d'enjeux politiques.

La question de l'inéligibilitéa été posée dès le début de l'examen du décret-loi sur la loi électorale par Sahbi Atig, l'un des représentants d'Ennahdha. Il souhaite que les députés et les individus ayant exercé des responsabilités au sein de l'État et du Rassemblement constitutionnel démocratique (RCD - le parti du président déchu) au niveau national et local durant les cinq ou dix dernières années du régime Ben Ali ne soient pas autorisés à se présenter à l'élection de la future assemblée ${ }^{34}$.

La liste des personnes susceptibles d'être interdites de candidature est précisée au fur et à mesure des débats. Aux responsables du RCD et du gouvernement, sont ajoutés ceux qui ont fait l'apologie du président Ben Ali et l'ont appelé à se présenter pour un nouveau mandat en 2014 (les mounachidin).

La rédaction finale du texte se rapportant à l'exclusion, présentée par le comité des experts, suscite des réactions au sein de l'Instance. En effet, l'article 15 du projet de décret-loi dispose, entre autres : «ne peut être candidat toute personne ayant exercé des responsabilités au gouvernement et dans les structures du RCD pendant les dix dernières années ${ }^{35}$. C'est précisément sur la mention des dix ans que les débats au sein de l'ISROR se cristallisent. Plusieurs intervenants accusent les experts et le président Ben Achour de vouloir imposer leur choix et exigent que l'on vote sur l'article 15 considérant précisément qu'il n'y a pas consensus au sein de l'Instance sur cette disposition.Le résultat final est sans appel puisque quasiment les trois-quarts des membres approuvent l'inéligibilité des individus ayant occupé de hautes fonctions politiques pendant les 23 ans du règne de Ben $\mathrm{Ali}^{36}$.

Le gouvernement exprime son désaccord sur ce dernier point considérant que prendre pour critère d'inéligibilité 23 ans reviendrait à écarter «des responsables réprimés par le régime de Ben Ali et d'autres connus par leur militantisme en faveur des droits de l'Homme, mais qui ont assumé des fonctions au début de l'ère Ben Ali » ${ }^{37}$. Pour éviter une confrontation entre l'ISROR et le gouvernement source de blocage du processus de «transition», le président de l'Instance se propose de négocier une nouvelle rédaction de l'article 15 avec le gouvernement : le nombre d'années ne serait pas précisé et le texte prévoirait que l'ISROR se chargerait d'établir la liste des responsabilités ${ }^{38}$.

Le gouvernement accepte de reprendre à son compte la formulation de l'Instance, alors qu'une commission s'attèle à définir le niveau de responsabilité au sein du RCD interdisant une candidature à l'ANC, ainsi qu'à établir la liste des mounachidin. In fine, bien qu'il ne soit pas fait mention de la durée comme critère d'inéligibilité, la limite de dix ans est appliquée et

\footnotetext{
${ }^{34}$ ISROR, Séance du 31 mars, p. 101.

${ }^{35}$ ISROR,Séance du 11 avril, p. 234.

${ }^{36} I d$. , p. 234

${ }^{37}$ R. Abdallah, «Interdiction pour les responsables du RCD de se porter candidat à l'Assemblée constituante. Le gouvernement provisoire réduit la période des 23 ans aux 10 dernières années (en arabe) », Al-Sabah, 27 février 2011.

${ }^{38}$ Le nouveau texte prévoit : « Ne peut être candidat toute personne ayant assumé une responsabilité au sein du gouvernement durant l'ère du président déchu à l'exception de celle qui n'ont pas appartenu au RCD ; toute personne ayant assumé une responsabilité au sein du RCD durant à l'ère du président déchu. Les responsabilités concernées seront fixées par décret sur proposition de l'ISROR ; toute personne ayant appelé le président déchu à être candidat pour un nouveau mandat en 2014. Une liste sera établie, à cet effet par l'ISROR ». ISROR,Séance du 5 mai, p. 344-345.
} 
environ 8000 individus sont interdits de candidature auxquels il faut ajouter 3000 mounachidin ${ }^{39}$.

La question de l'inéligibilité des responsables de l'ancien régime est remise à l'agenda politique par une partie des constituants, notamment les membres des partis de la Troïka ${ }^{40}$. Mais trois ans après le départ du président Ben Ali, la thématique de l'exclusion des anciens du RCD n'a plus la même signification.L'émergence en 2012 de NidaaTounes, parti qui s'est rapidement posé en challenger d'Ennahdha et dont le fondateur est un ancien haut responsable du RCD, a poussé à intervalles réguliersle mouvement islamiste et ses alliés à revendiquer l'exclusion des cadres du RCD de la vie politique.

En fait, la position de la direction d'Ennahdha a évolué sur cette question : après avoir soutenu en 2012 et 2013, la nécessité de voter un texte sur l'exclusion des responsables de l'ancien régime de la vie politique, intitulé « loi d'immunisation de la révolution », le chef du mouvement islamiste, Rached Ghannouchi, a déclaré, à plusieurs reprises, que son parti refusait l'adoption d'une proposition de loi aboutissant à une sanction politique contre une partie de la population. Le traumatisme toujours présent de la répression sous Ben Ali et la phobie d'un possible coup d'État en Tunisie, à l'image de celui mené en Egypte par l'armée contre les Frères musulmans, ont incité le dirigeant historique du mouvement à encourager les députés nahdhaouis à l'ANC et les militants du parti, " au compromis politique et au mariage de raison avec les ennemis d'hier ${ }^{41}$.

Une partie des constituants d'Ennahdha renâclant à suivre les directives du chef du parti,l'article 167 mentionnant, l'exclusion n'est rejetée que d'extrême justesse à une voix près, avant d'être définitivement repoussé le lendemain.

Le rejet par l'ANC de l'inéligibilité des responsables politiques de l'ancien régime consacre ainsi le retour sur la scène électorale de certains caciques du RCD qui se présenteront aux législatives et à la présidentielle de 2014.

L'application du principe de représentation-figuration à travers le choix du mode de scrutin proportionnel au plus fort reste sans seuil électoral, si ellea apportéun semblant de réponse à la question de la juste représentation, place désormais les gouvernants tunisiens devant la difficulté de trouver une majorité pour gouverner.

Mais force est de constater, dans un contexte de transition politique instable,que le choix de ce mode de scrutin a permis d'assurer un certain équilibre des forces entre la Troïka, la coalition gouvernementale, et son opposition. En empêchant un parti politique d'avoir la majorité absolue des sièges au sein de l'ANC, ila permis d'éviter que l'un des protagonistes de la scène politique ne soit tenté de porter atteinte aux intérêts vitaux de ses adversaires. Pour paraphraser les pères de l'analyse transitologique ${ }^{42}$, Ennahdha et les partis sécularistes ont dû passer des compromis de manière à rendre suffisamment compatibles leurs «offres politiques » et à permettre aux «règles de la compétition démocratique » de s'appliquer volens nolens.

\footnotetext{
39 M.Lieckefett, «La Haute Instance et les élections en Tunisie : du consensus au "pacte politique" », Confluences méditerranée, $\mathrm{n}^{\circ}$ 82, 2012, p. 138.

${ }^{40} \mathrm{C}$ 'est-à-dire le gouvernement de coalition dirigé par HamadiJebali et Ali Laarayedh, entre décembre 2011 et janvier 2014, rassemblant le Mouvement Ennahdha et de ses deux alliés « laïcs », le Congrès pour la République (CPR), le parti du président de la République transitoire, Moncef Marzouki et le Forum démocratique pour le travail et les libertés dirigé par le président de l'ANC.

${ }^{41}$ ICG, Élections en Tunisie: vieilles blessures, nouvelles craintes, update briefing, 19 décembre, 2014, $<$ http://www.crisisgroup.org $>$.

${ }^{42}$ G. O'Donnel et Ph. C. Schmitter, Transitions from Authoritarian Rule, Baltimore et Londres, The John Hopkins University Press, 1986, p. 37-38.
} 
$\mathrm{Au}$ final, la «rationalisation » du système électoral tunisien, dans l'optique de «l'obtention d'une majorité nécessaire à la gouvernabilité ${ }^{43}$ ne paraît réalisable que si les acteurs politiques sont assurés que l'application du principe majoritaire dans toute sa rigueur ne signifiera pas la négation des droits de la minorité.

${ }^{43}$ Y. Mény et M. Sadoun, op. cit., p. 10. 